\title{
Experimental and Analytical Pressure Characterization of a Rigid Flapping Wing for Ornithopter Development
}

\author{
Derrick Yeo ${ }^{*}$, Ella M. Atkins, ${ }^{\dagger}$ and Wei Shyy ${ }^{\ddagger}$ \\ University of Michigan, Ann Arbor, Michigan, 48109, U.S.A.
}

\begin{abstract}
Control of ornithopters is challenging due to the unsteady flow generated by the flapping wings. The research presented in this paper is aimed at developing a sensor-based control methodology. Specifically, a distributed pressure sensing system embedded in the flapping wing surfaces is utilized to provide real-time aerodynamic force estimates to facilitate the development of feedback for an ornithopter autopilot system. This paper describes the design, construction, and testing of a customized flapping mechanism with flat plate ornithopter wings featuring embedded pressure lines. Under varied flapping frequencies and free-stream conditions, cycle-averaged pressure distributions over the wings using external high-precision pressure sensors are presented.
\end{abstract}

$\begin{array}{ll}b & =\text { wingspan, } \mathrm{m} \\ c & =\text { chord, } \mathrm{m} \\ h & =\text { stroke amplitude, } \mathrm{m} \\ f & =\text { frequency of oscillation, } \mathrm{Hz} \\ U_{\infty} & =\text { free stream velocity, } \mathrm{m} / \mathrm{s} \\ R e & =\text { Reynolds number, } \mathrm{Re}=\frac{V_{\text {tip }} \cdot c}{v} \\ S t & =\text { Strouhal number, } S t=\frac{h \cdot f}{U_{\infty}} \\ V & =\text { velocity, } \mathrm{m} / \mathrm{s} \\ \beta & =\text { wing flapping angle, degrees }\end{array}$

\section{Nomenclature}

\section{Introduction}

$\mathrm{W}$ ith modern electronic payloads becoming more lightweight, unmanned aircraft systems (UAS) can now conduct demanding missions such as covert surveillance with very small payload capacities thus total vehicle weights. At these smaller scales, traditional fixed wing designs have considerable performance limitations. As the size of a fixed wing decreases, Reynolds number effects result in reduced lift and increased drag coefficients, requiring higher airspeeds that might not be practical for many UAS applications. Flapping wing systems, as evolved by nature, have been observed to enjoy aerodynamic benefits instead of penalties at these smaller scales ${ }^{1}$ allowing small flyers to thrive at small scales and lower airspeeds. These potential benefits are accompanied by considerable challenges such as highly unsteady aerodynamics and non-linear vehicle dynamics that make controlling flapping wing UAS difficult given the current decoupled longitudinal/lateral framework used in most applications. This paper presents the second part of an ongoing investigation of onboard sensing options to address the need for improved feedback for flight control. It focuses on the correlation between experimental pressure measurements and computational predictions to verify that the embedded pressure sensing system is able to provide accurate measurements. In earlier work, the authors have explored the use of high speed pressure sensing for flapping wing applications by building an instrumented flap stand that used commercially available ornithopter

\footnotetext{
* PhD student, Aerospace Engineering Dept, University of Michigan, Ann Arbor, MI, student member.

$\dagger$ Associate Professor, Aerospace Engineering Dept, University of Michigan, Ann Arbor, MI, Associate Fellow.

"Clarence L. "Kelly" Johnson Collegiate Professor, Department of Aerospace Engineering, University of Michigan; currently, Provost \& Chair Professor, Department of Mechanical Engineering, Hong Kong University of Science and Technology.
} 
mechanics. Motion kinematics with this commercial ornithopter were inconsistent, and did not provide an accurately-measurable flapping stroke. This meant that forming an in-depth computational case that matched experimental kinematics was not possible.

In this work, a robust flapping mechanism has been custom-designed and built to address these limitations by producing a consistent, easily quantifiable, and directly measurable wing beat. The new mechanics employ a transverse crank-rocker configuration used on existing ornithopter designs that produce an asymmetric flap stroke driving a pair of rigid Zimmerman wings with a planar airfoil section. Using a VICON motion capture system, measurements of the flapping stroke at different frequencies have been taken and compared to predicted kinematics to assess any potential deviations from the design stroke when the mechanism is flapping under high loads. High speed aerodynamic measurements, specifically an array of pressures across a flapping wing, have been taken both at a hover and in a free stream provided by a wind tunnel. The motion capture data compared well with computed values, showing less than $2 \%$ maximum error in flap angle. These computed values were then used as inputs to a discrete vortex method code. In this paper, some background is presented followed by an overview of the new flapping mechanics. The motion capture experiments are described and test results are presented.

\section{Background}

Previous experimental aerodynamics work range from measurements of live biological flyers ${ }^{2,3}$ and water tunnel studies of characteristic fluid flow fields ${ }^{4,5}$ to tethered flight of existing vehicles with sizes ranging from insect-like scales $^{6,7}$ to those of bird-type scales ${ }^{8}$. Computational developments have evolved along the lines of full NavierStokes CFD solvers and those of potential flow, discrete vortex type methods ${ }^{9,10}$. The thin membrane wings used by successful flapping wing flyers and the kinematics of the flapping process induces considerable coupling between fluid and structural dynamics in flapping wings, thus it is difficult to study one aspect without considering the other. Aerodynamic models must be coupled with structural solvers in order to describe the physics of a practical flapping wing. Researchers have coupled both CFD and discrete vortex methods with structural solvers ${ }^{11,12}$ that generate results to validate against existing experimental data.

Understanding the physics of the flapping dynamics is key to developing control algorithms. An extension of the standard aircraft model and time-averaged forces was used by Deng ${ }^{13,14}$ in developing a switching controller of an insect type flyer near hover. Bolender ${ }^{15}$ treated a flapping wing micro air vehicle (FWMAV) as a collection of four rigid bodies: two wings, body, and tail, and derived its equations of motion using Kane's equations. Researchers have used both experimental and computational results to formulate control strategies that aim to recreate a degree of the maneuverability of biological fliers. Doman ${ }^{16,17}$ used aerodynamic coefficients from oil tank experimental data to investigate split-cycle wing beat control with a non-linear dynamics model while Sun ${ }^{18}$ used CFD results in deriving the equations of motion of a FWMAV. The challenging non-linear dynamics have also led to the proposal of adaptive control methods such as neural networks ${ }^{19}$ by Chung and Dorothy for vehicle flight control.

Flapping wing vehicles have been built and tested by the engineering community, ranging in size from micro ${ }^{20,21}$ to meso scale $\sim 10 \mathrm{~cm}$ vehicles ${ }^{6,7,22}$, to tests done on larger scale vehicles ${ }^{23,24,25}$ with wingspans $\sim 1 \mathrm{~m}$. Autonomous ornithopter-scale flapping wing vehicle flights have been successfully conducted by researchers utilizing extended fixed wing autopilot formulations. Kranashita ${ }^{26}$ incorporated a simple Paparazzi (http://paparazzi.enac.fr/wiki/Main_Page) autopilot on a Cybird P2 ornithopter and demonstrated autonomous, waypoint tracking flight. More recently, Lee et $\mathrm{al}^{25}$ designed and built flapping wing platform SF-2 which flew autonomously with a more complete inertial measurement sensor suite.

Most current flapping wing vehicles rely on moving surfaces adapted from traditional aircraft designs for attitude control. A single tail surface typically provides pitch and directional control and the main wings are not actuated beyond their fixed flapping stroke. ${ }^{9}$ While such methods have flown on a variety of remotely piloted vehicles, both for recreational and research applications ${ }^{6,7,24,25,26}$ they fail to afford ornithopters the agility of fixed or rotary wing vehicles. Even the Festo Seagull ${ }^{27}$ which utilizes the most advanced wing actuation to date, cannot mimic the full capabilities of its biological namesake. Incorporating attitude control actuation on the moving wings of an ornithopter presents even more challenges due to constantly changing forces and inherently non-linear aerodynamics, but offer the possibility of aerobatic performance. Kranashita ${ }^{26}$ notes that a lack of accurate 
aerodynamic models of instantaneous aerodynamic forces on flapping wings remains the main obstacle for a formal mathematical analysis of flight dynamics and controls.

Wing flexibility has a significant influence on the aerodynamic performance of a flapping wing ${ }^{1,28,29}$ and plays a major factor in practical flight vehicles. However, with rigid wings, the number of relevant factors is smaller and the kinematics can be more accurately quantified using existing test methods, while still allowing insight into how pressure-based experimental results may support the development of reduced order models for design and control. Pressure measurements have been used previously in this context. In purely lab-based experimental work, Hilaire and Carta $^{30}$ took pressure measurements over a range of oscillating wings with a symmetrical airfoil with different planforms. Green and Smits ${ }^{31}$ studied pressure distribution over a 3D, 2 degree of freedom foil oscillating in a water stream and formulated a new propulsion scaling based on pressure. On UAS platforms, the authors ${ }^{32}$ have implemented a pressure based aerodynamic sensing system and integrated its data into a level-flight to hover transition guidance law. In a collaborative effort, an AFOSR MURI group ${ }^{33}$ has developed micro-tuft type MEMS sensors for flow direction and magnitude detection on a fixed-wing UAS. Most recently, researchers in Tokyo ${ }^{34}$ has demonstrated the near term potential of this sensing strategy by flight testing an insect-scale flyer with integrated pressure sensors in its flexible wing membranes.

In our previous work, a platform for the wind tunnel testing of flapping wing vehicles that enables high speed pressure sensing over the wings and force-torque measurements was developed by the authors. ${ }^{37}$ Preliminary pressure data was presented that served as a proof of concept for pressure based aerodynamic sensing. Two sets of rigid test wings were instrumented by installing pressure ports at locations that served to discretize the wings surface and provide an experimental 'pressure map'. Pressure histories over averaged flap cycles corresponded well to predictions made using simple, quasi-steady panel method codes, but more quantitative comparisons were not possible due to erratic experimental wing kinematics.

The research presented in this paper extends our previous work by focusing on enabling quantitative comparisons of pressure measurements and computed pressure predictions to provide a more quantitative verification of the proposed aerodynamic measurement scheme. In order to achieve this, new mechanics had to be designed, built and tested. The new design incorporates a new position encoding system and includes a custom set of flap electronics with a built-in flapping frequency controller. In addition to an updated wing encoding system, motion capture data was acquired to provide an additional, sub-millimeter accurate assessment of the consistency of the mechanics wing rigidity across a range of flapping frequencies. In the following sections, we describe the current flap-stand configuration, the current flapping mechanism design, sensor hardware, VICON test results and flap test results.

\section{Improved Flap Stand Configuration}

A custom flap stand was previously developed by the authors to take synchronized, high speed pressure, forcetorque (FT) and wing location readings during a wind tunnel test ${ }^{37}$. Primary instrumentation includes a bank of low pressure MEMS pressure sensors and a six-axis force-torque (FT) sensor. A schematic of the full flap stand is shown in the figure below. The test model, FT sensor, and a pitot probe are supported by an adjustable main arm to accommodate different wind tunnel tests. Both a hall-effect encoder integrated into the current flap mechanics and infra-red distance sensors are used to provide wing position data. Pressure lines and electrical wiring are routed to two aerodynamic shells located behind the stand which house the embedded computer, data acquisition hardware and pressure sensors. For more details see ref. 37. 


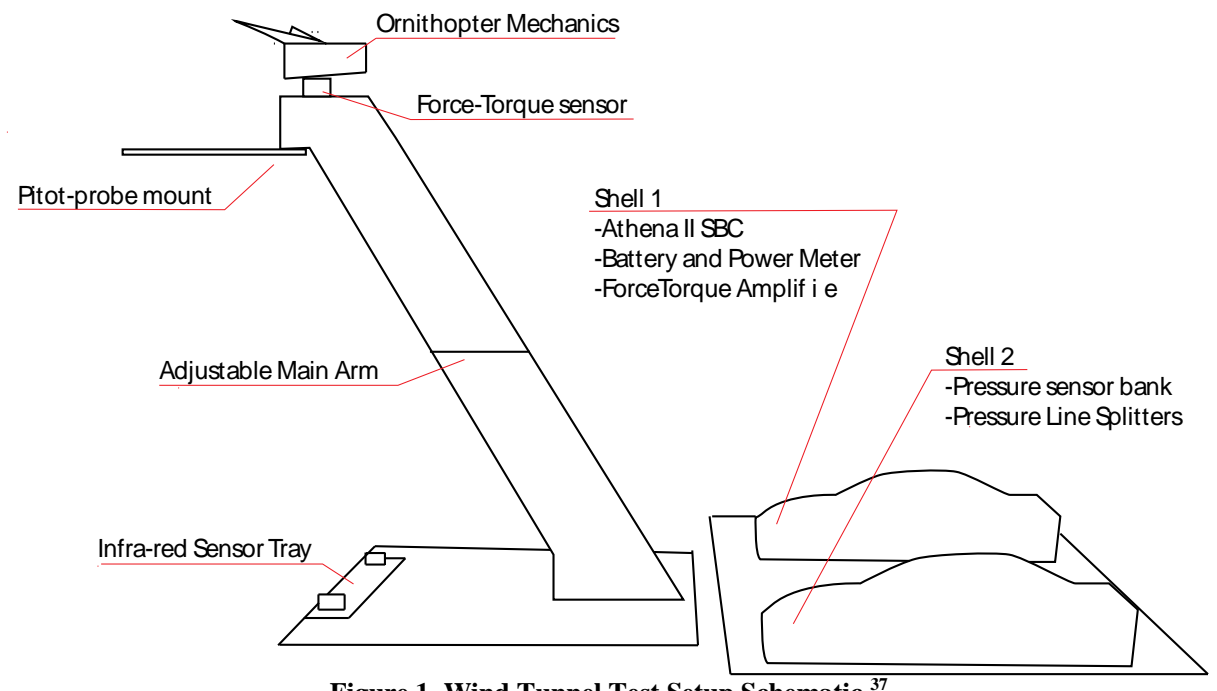

Figure 1. Wind Tunnel Test Setup Schematic ${ }^{37}$

Since the focus of this study was on pressure characterization, the FT sensor was removed and replaced with a fairing and a normal mount to eliminate the possibility of damage to the costly FT sensor during tests. The flapstand has been upgraded with a set of embedded electronics that control the flapping frequency of the test mechanics using a closed loop integral controller and also provides a pre-processed magnetic encoder signal to the PC104based data acquisition computer. A more detailed description of the flap governor is given in the mechanical design section.

\section{Mechanical Design}

The first generation flapping mechanism was taken from an actual Cybird flight vehicle ${ }^{37}$ and suffered from a fair amount of mechanical free play in both the main wing hinges, as well as backlash in the transmission. This caused wing kinematics to be inconsistent between different test conditions, limiting the ability to compare test and analysis results. Furthermore, the axially oriented transmission and crank arm produced a small phase difference between left-right wing strokes. These observations were consistent with those previously made by Hong and Altman ${ }^{36}$ who also used the Cybird mechanism to flap flexible in-house wings and quantify the lift generated by the simplest practical flapping kinematics. As a commercial alternative was not available to the authors, a revised set of mechanics was designed and built to address both these issues using sturdier parts, and a more rigid triple deck chassis was built with fiberglass plates and steel spacers. A transverse crank configuration used successfully by the hobby industry was adopted. Two sets of main wing bearings are included to support the main root of the wing to avoid torsional deflection along the feathering axis during flapping.

The current stage of this research requires consistent and repeatable mechanics for experimental testing, but with an ultimate goal of creating a flying platform. To facilitate this future purpose, the authors studied wing kinematics that were practical for ornithopter flight. The four-bar crank configuration is sturdy and already flight-proven in existing ornithopter designs. A flapping stroke similar to current ornithopters was realized in this work. It can be adjusted for amplitude and flap angle through the final linkage geometry. The transmission was designed to take advantage of high quality electric motor systems readily available for the hobby industry. The spur gear of the first reduction stage was chosen to mesh with Radio Control hobby helicopter 48-pitch pinion gears common for applications of this size. A six tooth 32-pitch pinion rod was interfaced with this spur gear and used to drive the final crank assembly that also accommodates a pair of magnets for wing position feedback. 


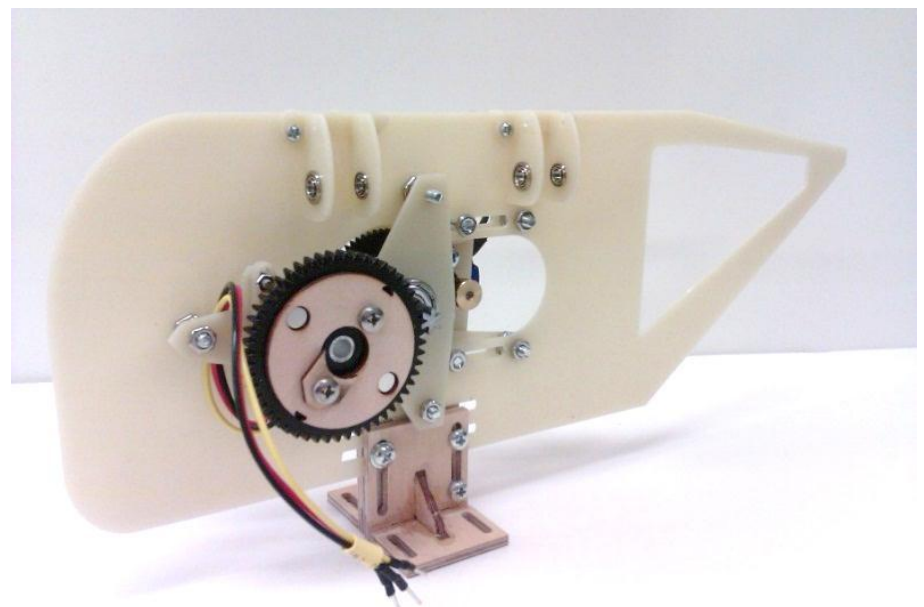

Figure 2. Flapping Transmission with Integrated Encoder System

Apker $^{38}$ found that using a brushed DC motor with no feedback control resulted in slightly variable flap frequencies. This is most likely caused by motor temperature changes during a test run. Such flap frequency variations would cause problems in pressure measurement consistency when acquiring data over an extended period of time. The new design presented in this paper also includes a custom-built magnetic encoder system and feedback controller to consistently regulate flap frequency. The magnets located on the final gear are detected by a hall-effect sensor and can be calibrated for crank offset by adjusting its mounting location. An embedded Atmel microcontroller conditions the signal and creates a logical change at two equally-spaced intervals during the flap stroke. The synchronization signal from the magnetic encoder combined with distance readings from a set of infrared range sensors to provide wing position data during experimental runs. The magnetic encoder system also provides feedback for a closed loop motor rpm controller that has been created for the test setup. The standalone device accepts user commands using a remote control to maintain the commanded reference frequency using an integralcontrol algorithm developed for this experiment. A schematic of this system is presented in Figure 3 below.

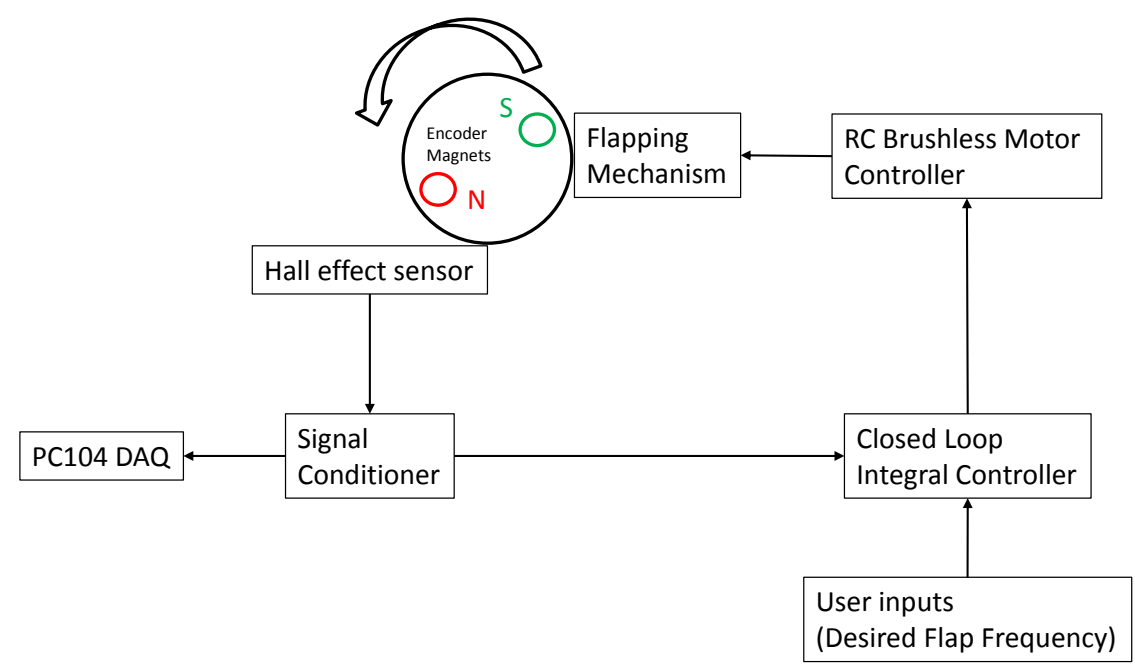

Figure 3: Flap Encoder and Embedded Flap Frequency Controller Schematic.

\section{Wing design and Pressure Port Location}

Using a technique previously developed by the authors, ${ }^{37}$ small pressure lines have been embedded into laminated balsa sheet wings with a Zimmerman planform. The wings have a planar cross section that has a thickness of $4 \%$ of the root chord. When mounted on the flapping mechanics, these wings have a plunge amplitude $h$ of $157 \mathrm{~mm}$. The 
pressure ports depicted in Figure 4 are connected to a bank of pressure sensors located on the flap stand. The sensors used were 0.25Inch-D4v differential sensors manufactured by All Sensors Corporation. These sensors provide high speed differential pressure measurements across the wing, allowing accurate estimates of aerodynamic forces normal to the wing.

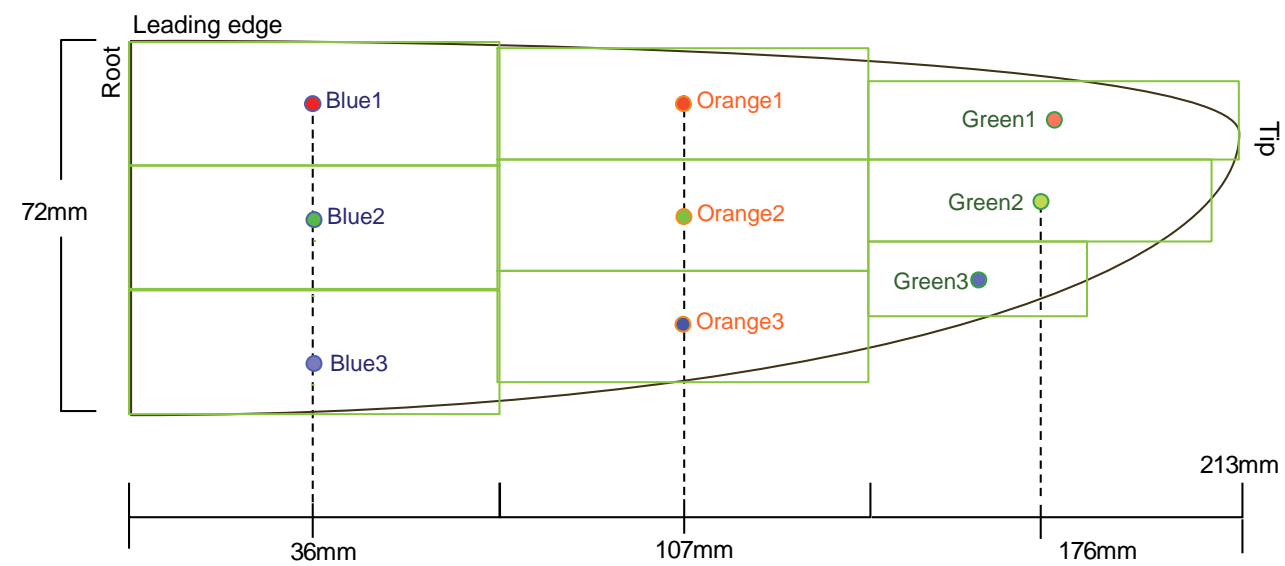

Figure 4: Wing Overview and Pressure Port Locations

When using the previous set of Cybird mechanics, it was found that the wing mounting system allowed a nontrivial amount of free-play along the feathering axis. This introduced an additional degree of freedom in the flap stroke which was neither easily-controlled nor easily-measured. To prevent this, the wings were designed to be hinged at fore and aft bearing holders on the flapping mechanism. The wing roots were constructed from harder basswood and designed to interlock with the main balsa wings to reinforce the wings along the feathering axis and eliminate unwanted wing twist as a result of actuating them from the leading edge. A sample of this style of construction is shown in the figure below.

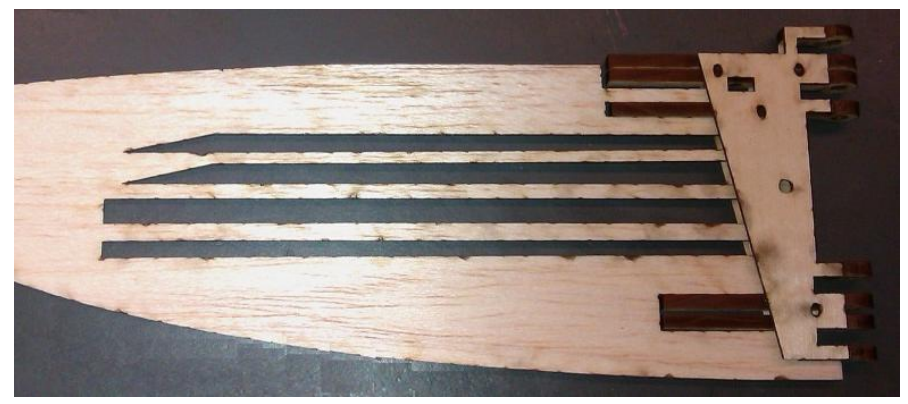

Figure 5: Example of The Wing Root Construction Technique Employed to Prevent Unwanted Wing Twist

\section{VICON Motion Capture Experimental Procedure and Results}

As described above, to ensure that a valid corresponding analytical simulation is used, it is critical that the flap kinematics be tested for stroke accuracy and repeatability. The existing infrared (IR) wing position sensors give relatively noisy measurements thus are not capable of fully-validating motion kinematics. The VICON motion capture system represents a more accurate alternate source of wing position data. Preliminary testing was carried out in a VICON environment from Michigan's Aerospace Robotics and Control Lab using large reflective targets, the flapping mechanism from Figure 2, and a pair of balsa wings with dimensions from Figure 4. VICON target locations are shown below in Figure 6. 


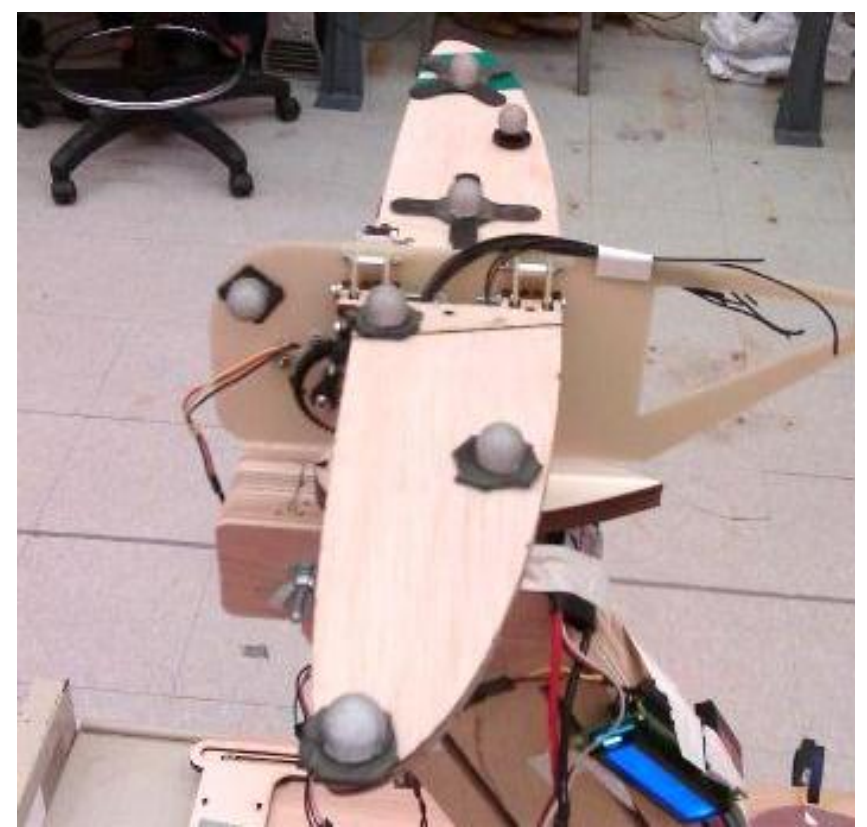

Figure 6: Flap Mechanics with Large VICON Targets

The main objective of this test was to confirm that the flap kinematics did not change appreciably at test frequencies between $1 \mathrm{~Hz}$ and $4 \mathrm{~Hz}$ and that the rigid wings did not deform when placed under load. For a preliminary test set, seven markers were used and their trajectories in space during a flap stroke are compared across a range of flapping frequencies. The underlying reasoning is that if marker trajectories were the same at $4.3 \mathrm{~Hz}$ as they were at $0.3 \mathrm{~Hz}$, this would indicate the flap mechanics remained rigid and consistent throughout the anticipated test frequency range. From the results, the mechanics and wing configuration tested were estimated to be consistent within $2 \%$ (by flapping angle) across the range of required flapping frequencies. Testing between $0.0 \mathrm{~Hz}$ and $1.3 \mathrm{~Hz}$ was not possible with the current power configuration as the drive motor and motor controller combination loses commutation when the mechanics are operated below $1.3 \mathrm{~Hz}$. However, it is unlikely that the mechanics will malfunction between $0.0 \mathrm{~Hz}$ to $1.3 \mathrm{~Hz}$ while being able to perform correctly between $1.3 \mathrm{~Hz}$ and $4.3 \mathrm{~Hz}$, the targeted flapping frequency range.

\section{Repeatability and Consistency under Load}

Ornithopter flapping stroke repeatability and consistency was evaluated using the VICON motion capture system with multiple visual markers placed on each wing. These 'standard' VICON markers weighed approximately 3 grams each and resulted in wing weights of approximately 20 grams. This meant VICON wing weights of approximately $140 \%$ of the instrumented test wings. Figures 7 through 9 show displacement trends over multiple flapping cycles for the two wings, where coordinate $\mathrm{X}$ represents fore-aft motion, $\mathrm{Y}$ represents lateral motion, and $\mathrm{Z}$ represents vertical motion. Collected data shows that marker paths do indeed match within the target frequency range. Overall $\mathrm{YZ}$ trajectories of the outboard wing markers are shown below for flap frequencies between $1.3 \mathrm{~Hz}$ and $4.3 \mathrm{~Hz}$. Dots of different colors represent paths taken at different flap frequencies and units are in $\mathrm{mm}$. These plots show raw data from complete flap stroke time histories, not averaged flap stroke histories to highlight any inconsistency in mechanism motion or measurement. 


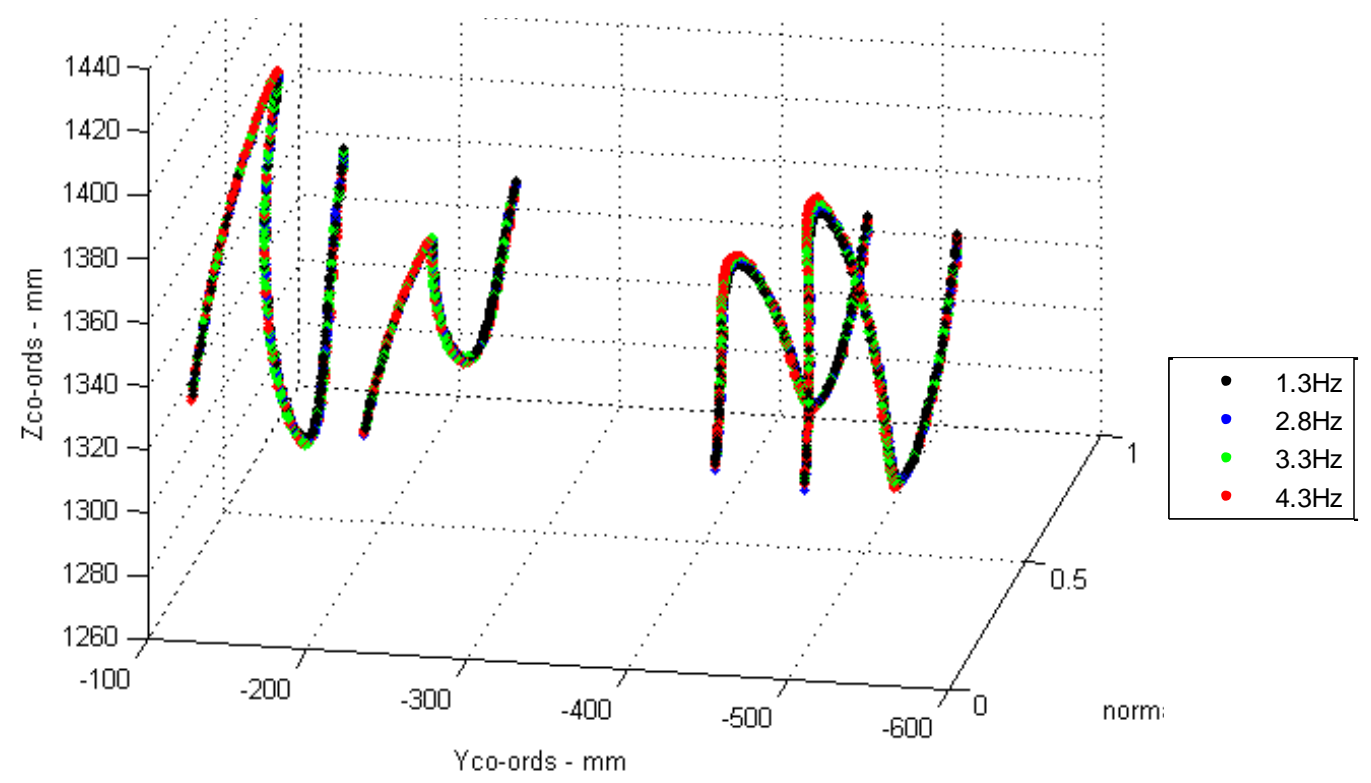

Figure 7: Wing Marker YZ paths at different flap frequencies

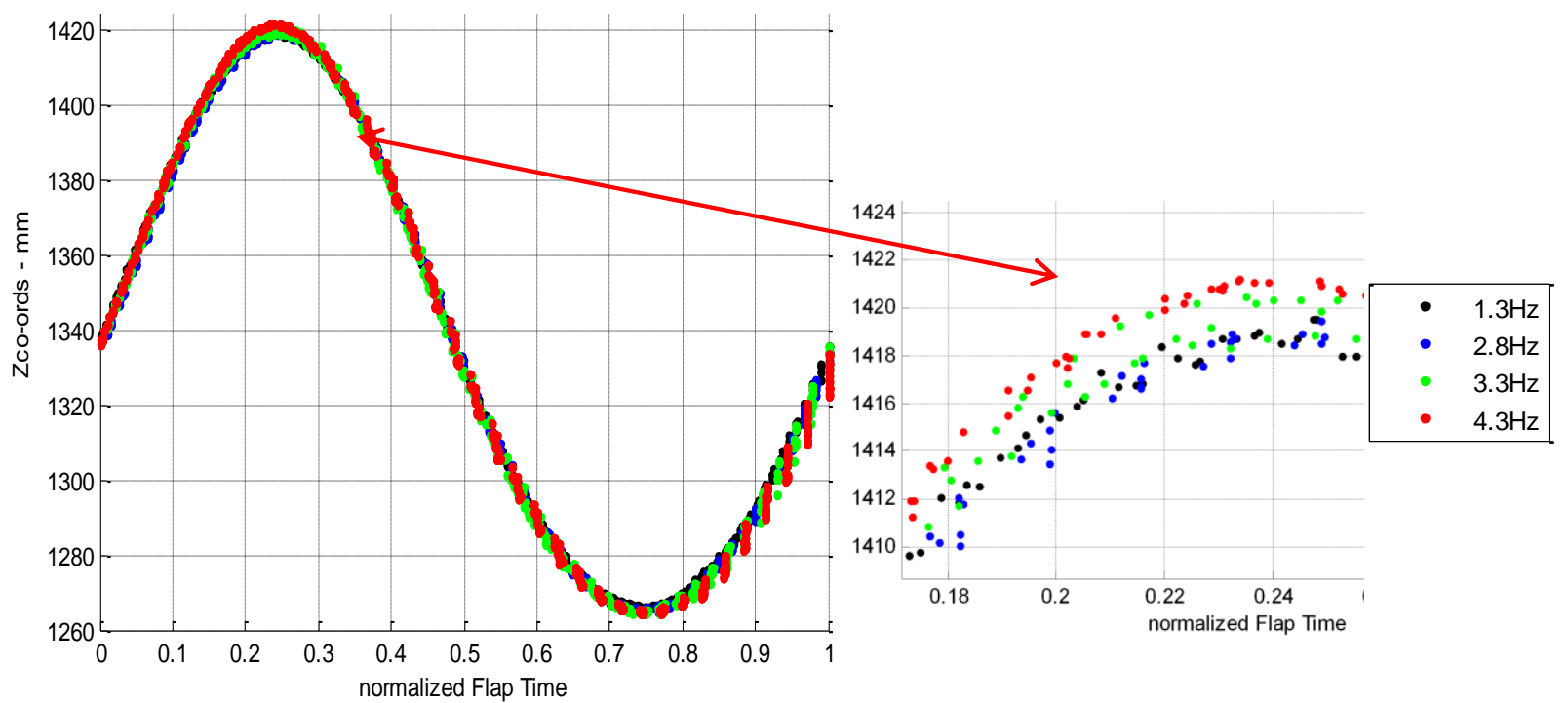

Figure 8: Port Wing Tip Marker Z paths at Operational Frequencies with Expanded View of Maximum Deviation in the Stroke.

From compiled VICON data, the maximum percentage error calculated by taking the largest encountered deviation from the baseline and normalizing by the total stroke amplitude. The maximum error from the above data set was found to occur at the top of the upstroke during the $4.3 \mathrm{~Hz}$ run, potentially due to some flexibility in the wings that are about overloaded by the VICON targets. This maximum error was computed to be less than $3 \%$ of the total amplitude.

\section{Absence of Unintended Deformation along The Feathering Axis}

Another concern was wing root deformation along the feathering axis introducing a pitch motion, as was encountered with previous mechanics. To estimate the severity of this kind of deformation in the current mechanics, the change in phase difference between fore and aft VICON markers on the wings were compared at multiple flapping frequencies. In a stroke where only the flapping angle changes, the phase difference between fore and aft

8 of 18

American Institute of Aeronautics and Astronautics 
markers should remain constant. These tests were run up to a higher frequency of $4.3 \mathrm{~Hz}$ to check for deflection along this axis. In Figure 9, Z-axis paths for fore-aft markers do not deviate in phase with increasing frequency, suggesting that changes in phase difference is negligible going from 1.3 to $4.3 \mathrm{~Hz}$.

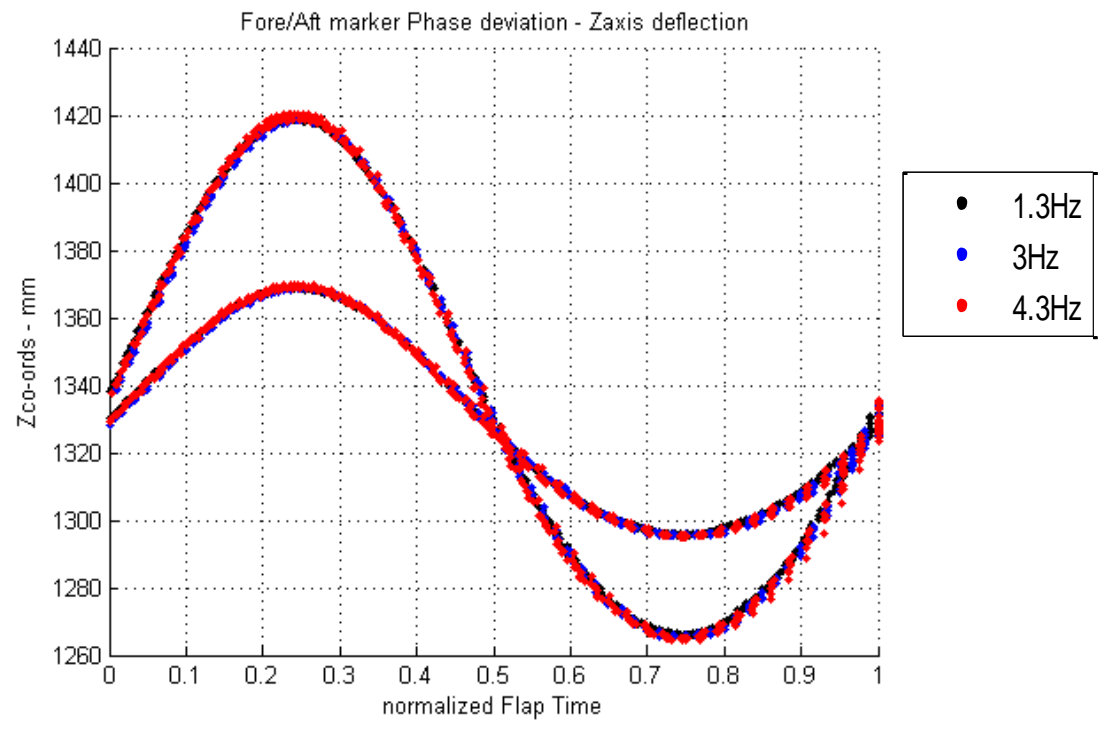

Figure 9: Port Wing Fore/Aft Marker Phase Difference Remains Constant Between 1.3Hz and 4.3Hz

\section{Experimental Validation of Linkage Solver}

During the mechanical design process, a 3D linkage code was written to provide wing flap angle as a function of crank position. This code was used for initial sizing of the components and during simulations it was used to predict actual wing kinematics produced by the final linkage geometry. A sample output of the $3 \mathrm{D}$ linkage solver is shown below in Figure 10.
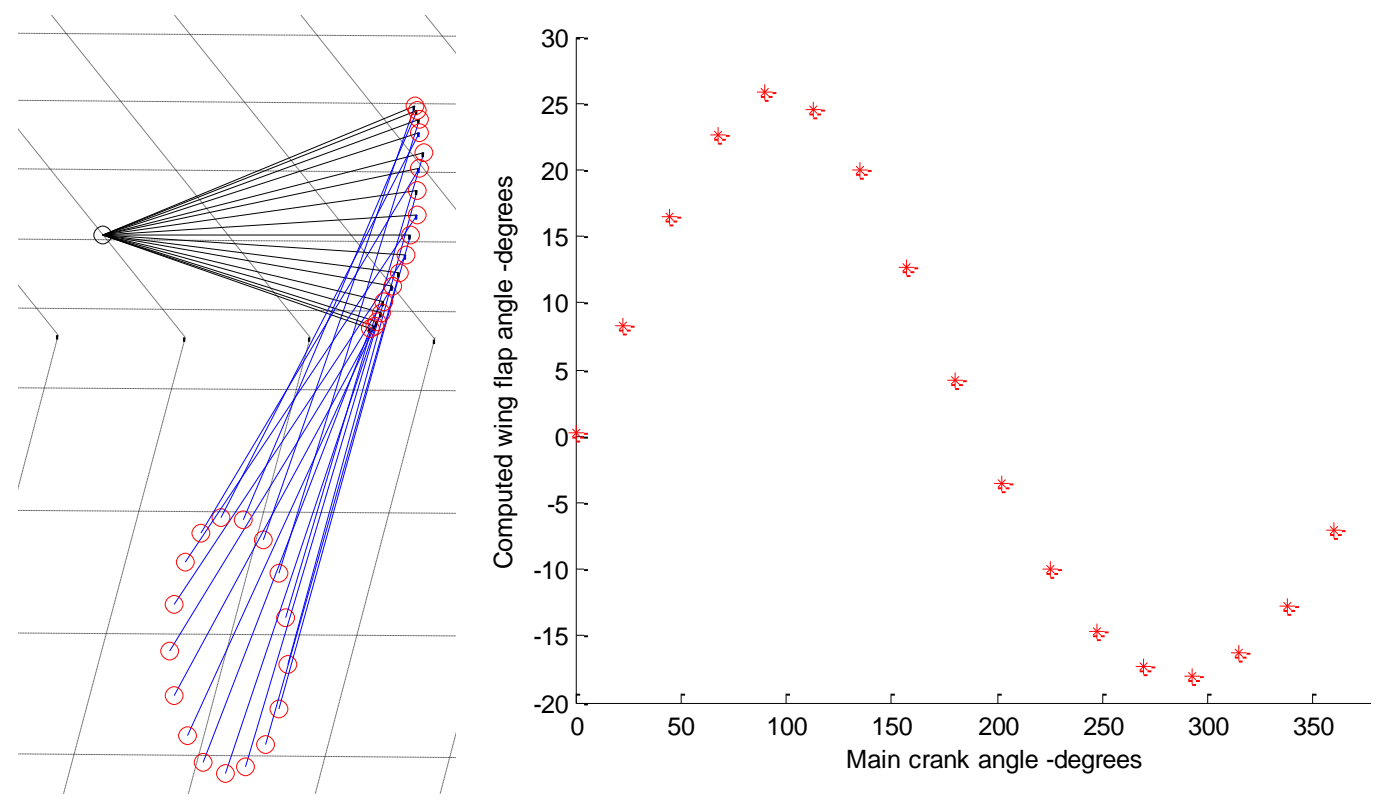

Figure 10: Sample Linkage Solver Output with 17 Crank Steps for Clarity. 3D View Showing Crank Positions with Red Dots and Wing Positions with Black Lines (Left). Corresponding Computed Wing Flap Angle $\beta$ Output at Given Crank Positions (Right). 
Figure 11 shows a comparison of compiled flap angle histories calculated using VICON marker position data and the output from the linkage solver. This comparison experimentally validates the kinematics solver over a stroke, and the assumption that crank rotation rate is constant throughout the flap cycle. On the previous Cybird mechanics, the transmission could not produce sufficient torque, resulting in an upstroke that was significantly slower than the down-stroke due to the mechanics struggling to lift the wings.

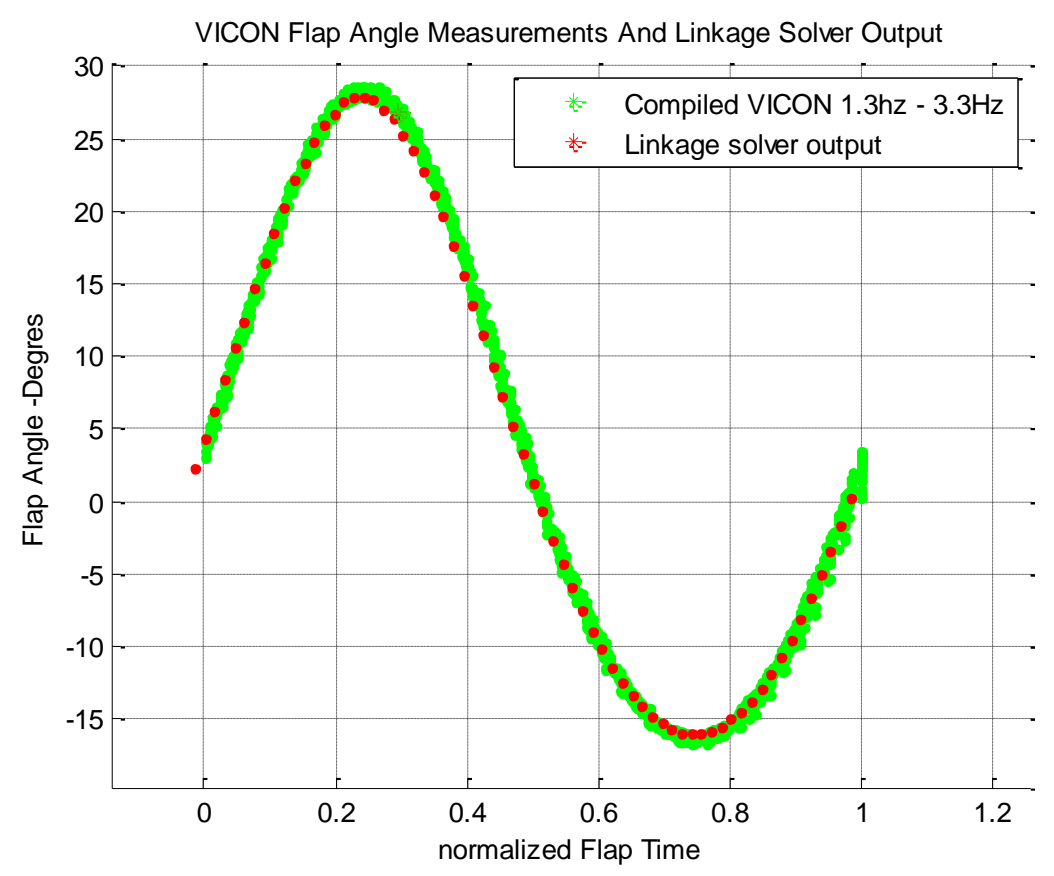

Figure 11: VICON and 3D Linkage Solver Stroke Comparison

As can be seen above in Figure 11, predictions and VICON measurements match. Again, the maximum error is considered. When compared to the baseline $1.3 \mathrm{~Hz}$ VICON run, the maximum measured error encountered at the top was found to be $\sim 0.3$ degrees, or less than $1 \%$ of full angular deflection. This consistency in phase indicates there is no appreciable loss of actuation rate on the upstrokes and that the transmission in the current mechanism produces sufficient torque for the system.

\section{IR System Calibration and Error Characterization}

The VICON system cannot be used in the wind tunnel due to tunnel volume and visibility constraints. The flap stand IR sensor system was therefore used instead. The validated flapping kinematics simulation can be used to assess the quality of the IR data. While highly accurate wing position data is not required, the IR system is useful when aligning test data with an analytical flap angle history. Figure 12 shows a comparison between the kinematics solver and raw $800 \mathrm{~Hz}$ IR data. The initial geometry used to determine flap angle was measured between wing root and the center of the sensor lenses. This was an initial value as the actual focal point of the IR beam on the wing's span was unknown. Since the mechanics were validated with the VICON system, this value for IR beam focus point and an angle offset term was then adjusted until IR data best matched the known kinematics simulation results. 


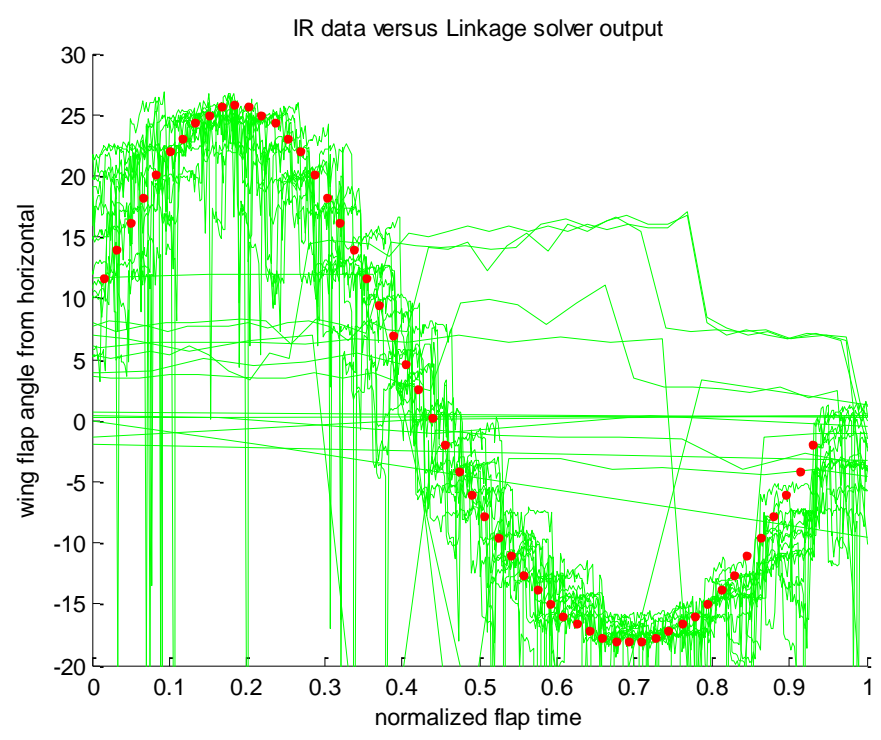

Figure 12: Adjusted Raw IR and 3D Linkage Solver Stroke Comparison

These adjustments of the IR sensor parameters allowed ensemble averaged IR data to be easily synchronized with a computed angular history. While less accurate when compared to measurements taken with the VICON system, the IR data provides sufficient position information to determine wing flap cycle stage. This data is used to account for small systematic offsets in the magnetic encoder system and provides a secondary source of wing position data.

\section{Data Processing Methodology}

The flap mechanics and sensor configuration allowed a straightforward process for compiling test data and constructing characteristic pressure histories for each test case. This is described below.

\section{Magnetic Encoder Signal}

A magnetic encoder system was integrated into the mechanics to aid flap cycle identification within data sets. The system provided consistent stroke encoding. A sample output is shown below in Figure 13.

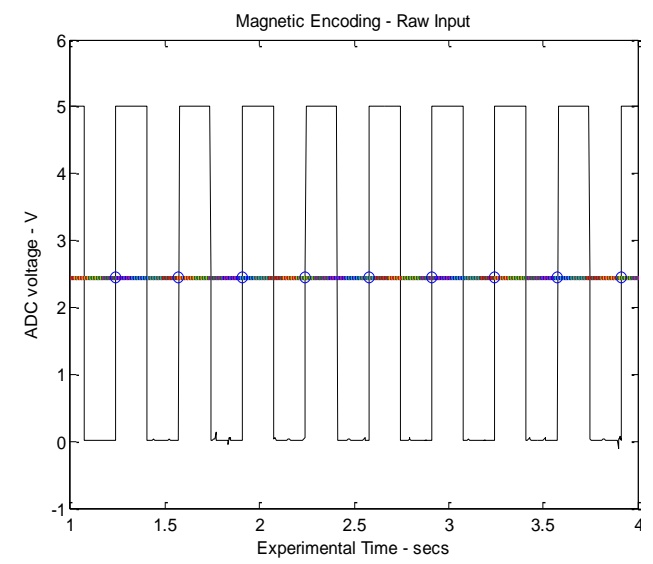

Figure 13: Raw Magnetic Encoder Data for Flap Cycle Identification

11 of 18

American Institute of Aeronautics and Astronautics 
The square wave encoder signal allows flap stroke identification to be performed on raw data and has been shown to provide consistent flap timing information. During post processing, this signal was used to identify individual flap strokes in an accurate and consistent manner. This allowed an ensemble averaging technique that accounts for slight phase time offsets between individual flap strokes and was amenable to an error characterization and the calculation of $95 \%$ confidence intervals (CIs) in both pressure and stroke time. The process begins by normalizing all measured flaps and then generates a two-dimensional 'point cloud'. The compiled data is searched for values that can be associated with a single time in an average stroke. These points are accumulated as the sample population for one averaged cycle measurement, and the statistics for both pressure and time were determined using a $95 \%$ confidence interval (CI) assuming a normal distribution.

A sample output is shown below. For illustrative purposes a coarse 5 point cycle average is shown in Figure 14. The 'population' of each data point is marked green. The $95 \% \mathrm{CI}$ for time is denoted by the red markers, and the $95 \% \mathrm{CI}$ for pressure lies between the blue markers. A more practical, 50point cycle average is then shown in Figure 15.

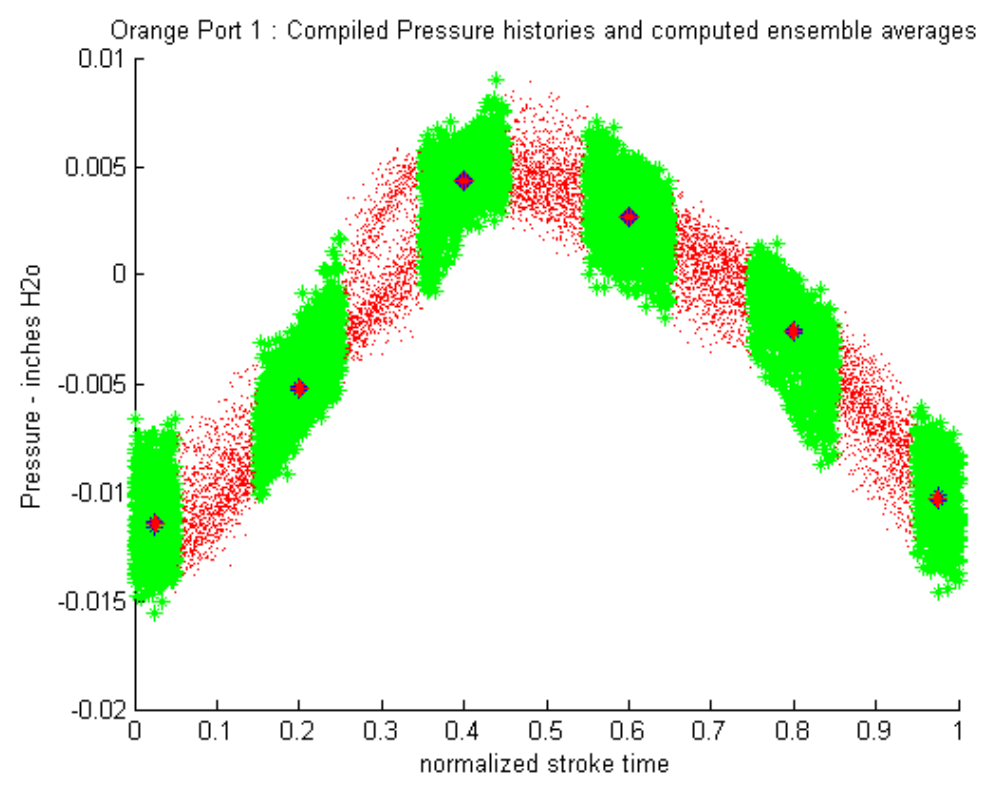

Figure 14: 'Point-Cloud' Ensemble averaging, coarse illustration. Green points denote sample populations, red markers denote $95 \%$ CI in time and blue markers bracket $95 \%$ CI for pressure.

12 of 18

American Institute of Aeronautics and Astronautics 


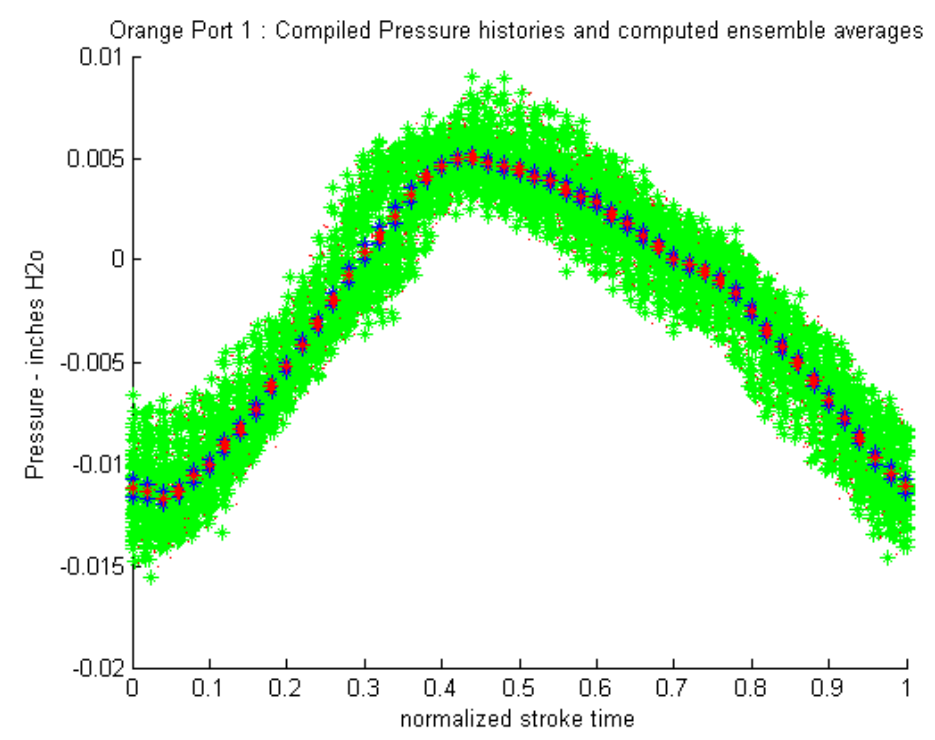

Figure 15: 'Point-Cloud' Ensemble Averaging. Green Points Denote Sample Populations, Red Markers Denote 95\% CI in Time and Blue Markers Bracket $95 \%$ CI for Pressure.

\section{Hover and Forward Flight Test Data}

With quantifiable kinematics, the pressure data collected can be compared with numerical results for the same conditions. To this end, a short series of flap tests were carried out to gather pressure data with the new mechanical setup. Both hover and forward flight cases were run and these are summarized in the tables below. Test names that are typed in bold are referenced below.

Table 1: $2^{\text {nd }}$ Generation Elliptical Wing Test Series, No Free-stream

\section{Hover}

\begin{tabular}{|l|l|l|l|}
\hline \multicolumn{4}{|l|}{ Flap Frequency } \\
\hline $2.1 \mathrm{hz}$ & $2.8 \mathrm{hz}$ & $3 \mathrm{hz}$ & $4.3 \mathrm{hz}$ \\
\hline testH1 & testH2 & testH3 & testH3b \\
\hline
\end{tabular}

Table 2: $2^{\text {nd }}$ Generation Elliptical Wing Test Series, $2.9 \mathrm{~m} / \mathrm{s}$ Free-stream

\begin{tabular}{|l|c|c|c|c|c|}
\cline { 2 - 6 } \multicolumn{1}{c|}{ Forward Flight } & \multicolumn{5}{|c|}{ Approximate $\mathrm{St}$ number: } \\
\cline { 2 - 6 } \multicolumn{1}{c|}{} & $\mathrm{St}=0$ & $\mathrm{St}=0.13$ & $\mathrm{St}=0.17$ & $\mathrm{St}=0.19$ & $\mathrm{St}=0.21$ \\
\hline Vehicle Alpha $=0 \mathrm{deg}$ & testA & testB & testC & testD & testE \\
\hline
\end{tabular}

For the forward flight cases, flapping frequencies corresponding to lower Strouhal (St) numbers were chosen to facilitate future comparisons to unsteady panel results. In previous work ${ }^{37}$ it was noted that during a flap cycle at $S t$ $=0.21$, full separation at the leading edge did not appear to occur at the inboard chord section and only began to separate near the half-span. This was observed through pressure profiles indicating large leading edge pressure differences at the blue inboard ports and fully separated flow profiles at the yellow and green pressure ports ${ }^{37}$.

This represents an added challenge to running the unsteady panel code chosen for comparison as additional information is needed to determine where along the span the leading edge vortex remains stable. An unsteady panel $\operatorname{code}^{39}$ was chosen for this stage of the research because results can be quickly generated for different cases and different wing designs. A future comparison of results to a Navier-Stokes solution is also planned. 


\section{Hover}

Two no-free-stream hover cases with flapping frequencies of $2.1 \mathrm{~Hz}$ and $3 \mathrm{~Hz}$ were run. The Reynolds number $(\mathrm{Re})$ by tip velocity for these two cases was estimated to be between 12,000 and 14,000. The resulting pressure measurements are summarized in the plots below.
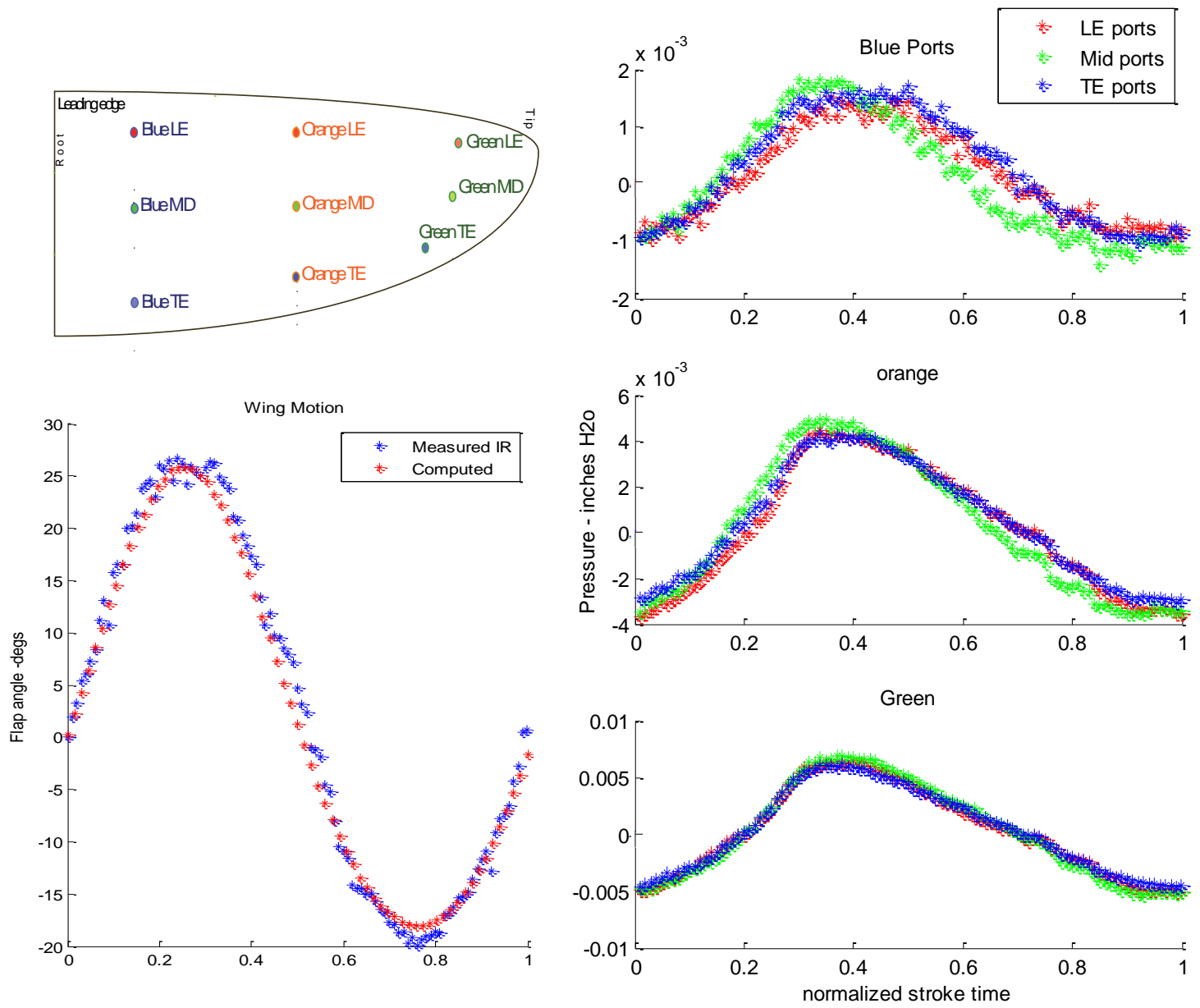

Figure 16: Data Overview for Hover at 2.1Hz: Flap Angle (Left) and Pressure Histories (Right) Blue Chord:17\% span; Orange: midspan; Green: $83 \%$ span.

At $2.1 \mathrm{~Hz}$, the pressure distribution across the wing suggests the flow is fully separated across the entire surface as can be expected in a hover situation. Pressure measurements at different ports along each chord show similar values of pressure and no indication of leading edge suction. Due to the asymmetric flap stroke, the pressure curves have a slight positive offset suggesting that a small amount of net lift is produced every cycle. A small plateau in pressure when the wing approaches the end of the downstroke can also be observed near normalized stroke times of 0.7. This is more apparent at a higher flapping frequency as shown in the $3 \mathrm{~Hz}$ case below.

14 of 18

American Institute of Aeronautics and Astronautics 

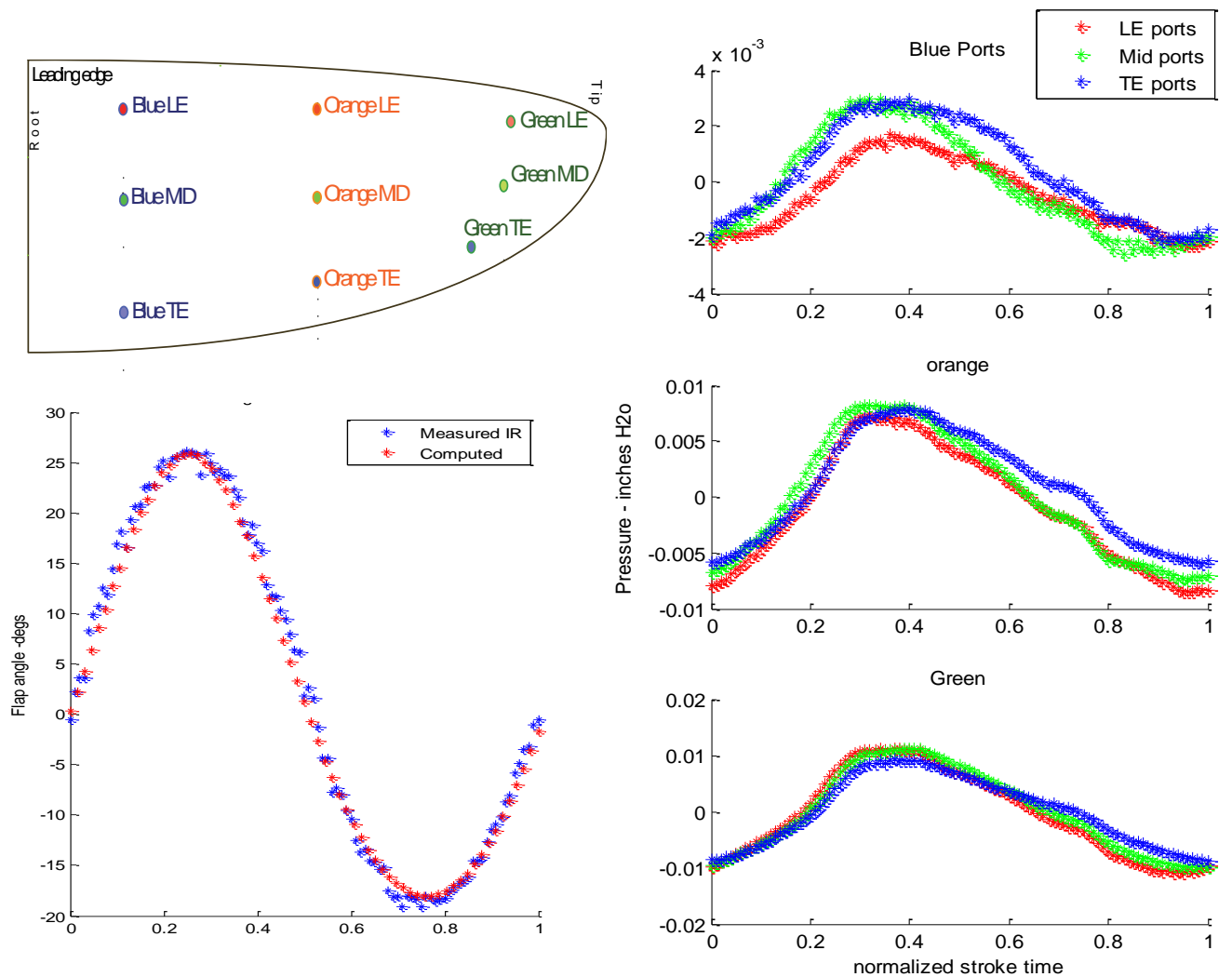

Figure 17: Data Overview for Hover at 3Hz. Blue Chord:17\% span; Orange: mid-span; Green: 83\% span.

This is likely due mainly to wing kinematics. When coming nearly to a standstill at the bottom of the flap stroke, the instantaneous velocity of the wing approaches zero. With no inflow incident on the wing, the resulting pressure difference at that point is close to zero.

\section{Forward Flight}

Wind tunnel testing was conducted in the UM $5 \mathrm{ft} . \times 7 \mathrm{ft}$. wind tunnel. The flap stand mounted in the test section is shown in Figure 18.

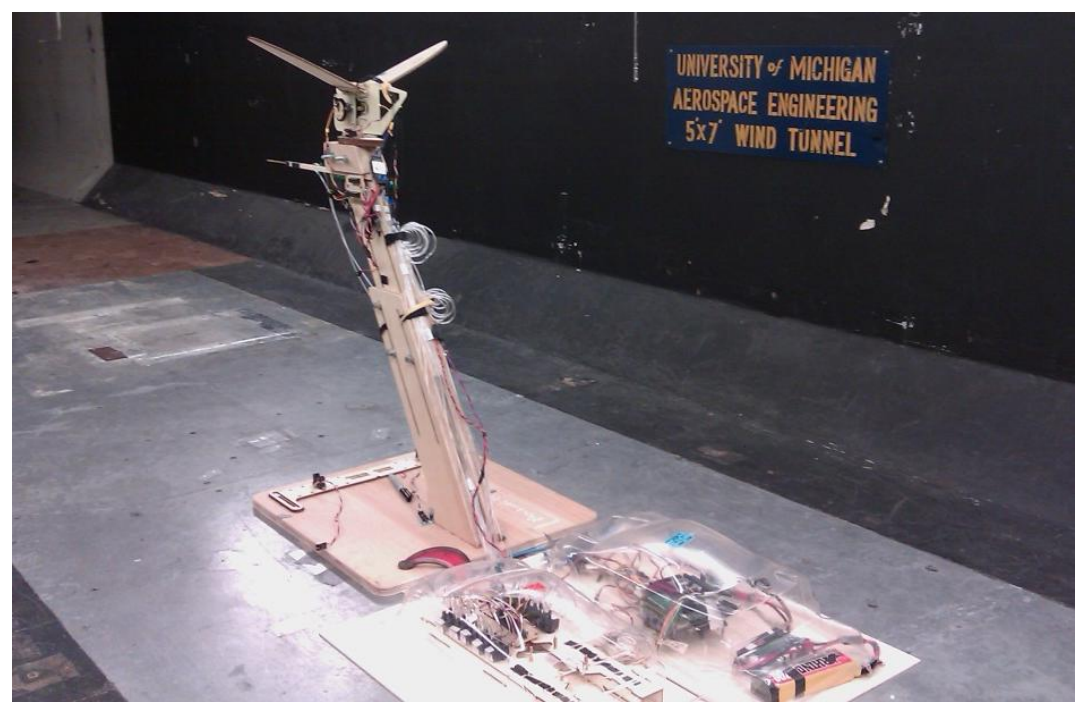

Figure 18: Wind Tunnel Test Setup

15 of 18

American Institute of Aeronautics and Astronautics 
The wind tunnel was run at a free stream of $2.9 \mathrm{~m} / \mathrm{s}$. At these speeds, the turbulent intensity of the wind tunnel was previously measured between $2-5 \%{ }^{37}$. Data was collected at $1 \mathrm{kHz}$ and for 8 seconds.
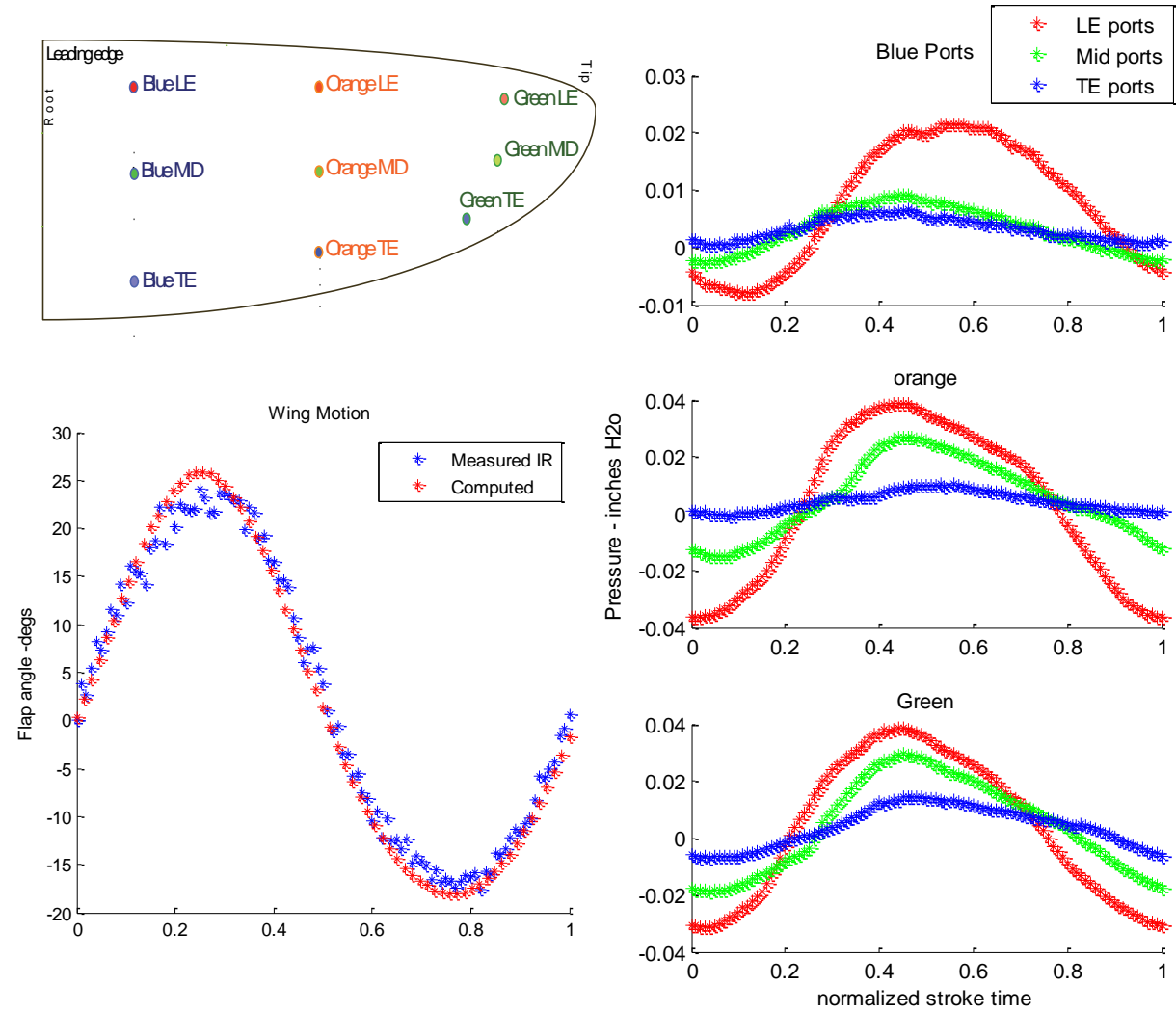

Figure 19: Data Overview for Forward Flight, 2.8m/s with a Flap Frequency of 3.3Hz. Blue Chord:17\% span; Orange: mid-span; Green: $83 \%$ span.

In a forward condition, the pressure measurements are significantly higher than those observed at hover. As can be seen from the data, a significant leading edge pressure difference is present at all chord locations throughout the stroke suggesting that the flow is separated at the leading edge. The observed enhanced lift generation near the wing tip and spanwise variation in pressure further indicate the 3D character of the flow field. The detailed interplay between the aerodynamic forces and the flow field will be explored using an unsteady panel code and a NavierStokes equation solver in future work.

\section{Conclusions and Future Work}

This paper has presented recent developments in an ongoing investigation of using pressure based sensing as feedback for ornithopter flight control. A custom designed and fabricated flapping mechanism was described along with a motion capture test series to validate the resulting wing beat kinematics. Hover and forward flight results are presented and a first order comparison to a quasi-steady panel calculation has been made for the forward flight case. Below are the major conclusions that can be drawn from the research presented.

- A university-designed alternative to commercial ornithopter mechanics has been constructed and determined to provide repeatable flap strokes on a test stand as validated with VICON tests and kinematic simulation. Maximum error for tip displacements did not exceed 3\% during an average stroke.

- Through a series of hover and forward tests, the mechanics and instrumentation presented have gathered consistent and reliable flap data at hover and in forward flight conditions. 
- A magnetic encoding strategy has been combined with simple data processing methods to enable high quality ensemble averaging with straightforward error analysis. Consistent wing position encoding coupled with an existing analytical kinematic model enables test data to be aligned with known stroke mechanics.

- For the wing geometry described, wind tunnel pressure data at lower St of 0.17 suggest leading edge separation across the span enhancing the lift generation.

With reliable wing kinematics and pressure measurements, the next step is to make comparisons between numerical solutions and pressure test data. After a formal verification of the pressure system is complete, force-torque measurements of the aerodynamic forces will be taken in order to form a working model relating real time pressure measurements to the forces generated. In order to achieve this, a multiphase wind-tunnel / vacuum-chamber test has been planned so that inertial and aerodynamic forces can be separated by subtracting inertial measurements taken in a wind tunnel from the total loads measured in a wind tunnel. Once a functional method of aerodynamic force estimation through pressure measurements is established, basic wind tunnel based control experiments are planned.

\section{Acknowledgements}

The authors would like to thank Professor Anouck Girard, Mr. Zahid Hassan, and the University of Michigan Aerial Robotics and Control Lab for facilitating the VICON experiments. The authors also thank Mr. Chris Chartier for providing wind tunnel support.

\section{References}

1 W.Shyy, Y. Lian, J. Tang, D. Viieru, and H. Liu, Aerodynamics of Low Reynolds Number Flyers, Cambridge University Press, 2007.

2 S. Walker, A. Thomas, G. Taylow, "Deformable Wing Kinematics in the Desert Locust: How and Why do Camber, Twist and Topography Vary Through the Stroke?” J R Soc Interface 2008; 6 (38): 735-747.

3 Wang H, Zeng L, Liu H, Yin C. Measuring wing kinematics, flight trajectory and body attitude during forward flight and turning maneuvers in dragonflies. J Exp Biol 2003; 206: 745-757.

4 D. Read, J. Hover, M. Triantafyllou, "Forces on Oscillation Foils for Propulsion and Maneuvering," J. Fluid Struct., vol. 17, pp. $163--93,2003$.

5 Y. Baik, J. Rausch, L. Bernal, and M. Ol, "Experimental Investigation of Pitching and lunging Airfoils at Reynolds Number between 1x10^4 and 6x10^4," Proc. 39th AIAA Fluid Dynamics Conference, San Antonio, Texas, June 22-25, 2009, 2009 , pp. AIAA-2009-4030.

6 D.Mueller, H.A. Bruck, S.K. Gupta, "Measurement of Thrust and Lift Forces Associated With Drag of Compliant Flapping Wing for Micro Air Vehicles Using a New Test Stand Design”, J. Experimental Mechanics. vol. 50, pp. 725 - 735, 2010

7 K. De Clercq, R. de Kat, B. Remes, B. van Oudheusden, and H. Bijl, "Flow Visualization and Force Measurements on a Hovering Flapping-Wing MAV 'DelFly II'," Proc. 39th AIAA Fluid Dynamics Conference, 22-25 June 2009, San Antonio, Texas. (AIAA-2009-4035)

8 J. Han, J. Lee, and D. Kim, “Bio-inspired Flapping UAV Design: A University Perspective,” SPIE, Vol. 7295, 2009.

9 S.Ansari, R.Zbibowski, and K.Knowles, "Non-linear Unsteady Aerodynamic Model for Insect-like Flapping wings in the Hover. Part 1" Proceedings of the Institution of Mechanical Engineers, Part G: Journal of Aerospace Engineering, vol. 220, no. 2, pp. 61-83, 2006.

10 D.J.Willis, J.Peraire and J.K.White, "FastAero - a Precorrected FFT - Fast Multipole Tree Steady and Unsteady Potential Flow Solver," presented at the SMA Symposium, Singapore 2005.

11 H. Aono, S.K Chimakurthi, P. Wu, E.Sällström B.K. Stanford, C.E.S. Cesnik, P. Ifju, L, Ukeiley, and W. Shyy, " A Computational and Experimental Study of Flexible Flapping Wing Aerodynamics," Proc. 48th AIAA Aerospace Sciences Meeting Including the New Horizons Forum and Aerospace Exposition, Orlando, Florida, (AIAA-2010-554).

12 A. Gogulapati and P. Friedmann, "Approximate Aerodynamic and Aeroelastic Modeling of Flapping Wings in Hover and Forward Flight," Proc. 52nd AIAA/ASME/ASCE/AHS/ASC Structures, Structural Dynamics and Materials Conference 19th AIAA/ASME/AHS Adaptive Structures Conference, Denver, CO, 2011. (AIAA-2011-2008)

13 X.Deng, L. Schenato, W. Wu, and S. Sastry, "Flapping Flight for Biomimetic Robot Insects: Part 1: System Modelling," IEEE Transactions on Robotics and Automation, Vol. 22, No.4, Aug. 2006, pp.776-788.

14 X.Deng, L. Schenato, W. Wu, and S. Sastry, "Flapping Flight for Biomimetic Robot Insects: Part 2: Flight Control Design," IEEE Transactions on Robotics and Automation, Vol. 22, No.4, Aug. 2006, pp.789-803.

17 of 18

American Institute of Aeronautics and Astronautics 
15 M. Bolender, "Rigid Multi-Body Equations-of-Motion for Flapping wing MAVs using Kane's Equations," Proc, AIAA Guidance Navigation and Control Conference, Chicago, IL 2009. (AIAA 2009-6158)

16 D. Doman, D. Oppenheimer, D. Sigthorrson, "Dynamics and Control of a Minimally Actuated Biomimetic Vehicle, Part1: Aerodynamic Model," Proc. AIAA Guidance Navigation and Control Conference, Chicago, IL 2009. (AIAA 2009-6160)

17 D. Doman, D. Oppenheimer, D. Sigthorrson, "Dynamics and Control of a Minimally Actuated Biomimetic Vehicle, Part1: Control," Proc. AIAA AIAA Guidance Navigation and Control Conference, Chicago, IL 2009. (AIAA 2009-6161)

18 M. Sun, J. Wang, and Y. Xiong, "Dynamic Flight Stability of Hovering Insects," Acta Mechanica Sinica, Vol. 23, No. 3, pp. 231-246, 2007

19 S. Chung and M. Dorothy, "Neurobiologically Inspired Control of Engineered Flapping Flight", J. Guidance Control and Dynamics Vol. 33 No.2, 2010

20 R. Wood, "The First Takeoff of a Biologically Inspired Robotic Insect" IEEE Transactions On Robotics, Vol.24, No.2, April 2008.

21 D. Dawson, " Repeatable Manufacture of Wings for Flapping Wing Micro Air Vehicles Using Microelectromechanical Systems (MEMS) Fabrication Techniques" Masters thesis, Air Force Institute of Technology, March 2011

22 D. Li, S. Guo, N. Matteo, D. Yang, “" Design, Experiment and Aerodynamic Calculation of a Flapping Wing Rotor Micro Aerial Vehicle", Proc. 52 ${ }^{\text {nd }}$ AIAA Structures, Structural Dynamics and Materials Conference, 4-7 April 2011, Denver Colorado (AIAA 2011-1988)

23 J. Grauer and J. Hubbard Jr. "Development of a Sensor Suite for a Flapping-Wing UAV Platform," Proc. 46 th $^{\text {AIAA }}$ Aerospace Sciences Meeting, January 2008. (AIAA 2008-224)

24 Z. Jackowski directed by R. Tedrake, Design and Construction of an Autonomous Ornithopter, MS Thesis, Massachusetts Institute of Technology, June 2009.

25 J. Lee, D. Lee and J. Han, "Ornithopter Attitude Estimation: Ground Test" World Automation Congress, pp. 1-6 19-23 September 2010

26 R. Kranashita, D. Silin, S. Shkarayev, "Flight Dynamics of Flapping-Wing Air Vehicle" Proc, $49^{\text {th }}$ AIAA Aerospace Sciences Meeting, January 2009 Reno, NV. (AIAA 2008-6698)

27 Festo (2011, Mar,) Festo. [Online]. http://www.festo.com/cms/en_corp/11369.htm

28 Agrawal A, Agrawal SK. Design of bio-inspired flexible wings for flapping-wing micro-sized air vehicle applications. $A d v$ Robotics 2009; 23(7-8): 979-1002.

29 W. Shyy, H. Aono, S. Chimakurthi, P. Trizilia, C.-K. Kang, C. Cesnik and H. Liu, " Recent progress in Flapping Wing Aerodynamics and Aeroelasticity," Prog. Aerospace Science., Vol.46, No.7 pp. 284-327, 2010

30 A. Hilairw and F. Carta, "Analysis of unswept and swept wing chordwise pressure data from an oscillating NACA 0012 airfoil experiment. NASA CR-3567, 1983

31 M. Green and A. Smits, "Effects of Three-Dimensionality on Thrust Production by a Pitching Panel", J. Fluid Mech. Vol.615 pp211-220, 2008

32 D. Yeo, J. Henderson, and E Atkins, "An Aerodynamic Data System for Small Hovering Fixed-Wing UAS," Proc. AIAA Guidance, Navigation, and Control Conference, 10-13 August 2009, Chicago Illinois. (AIAA 2009-5756)

33 Air Force Office of Scientific Research (AFOSR) Multidisciplinary University Research Initiative (MURI) AFOSR Grant FA9550-05-1-0411, [online] http://www.avocet.gatech.edu

34 H.Takahashi, Y.Aoyama, K.Ohsawa, H. Tanaka, E. Iwase, K. Matsumoto, I.Shimoyama, "Differential pressure measurement using a free flying insect-like ornithopter with a MEMs sensor" Bioinspiration and Biomimetics, vol. 5 no. 3, $036005,2010$.

35 D. Kim, H.Kim, J.Han, and K, Kwon. "Experimental Investigation on the Aerodynamic Characteristics of a Bio-mimetic Flapping Wing with Macro-Fiber-Composites". Journal of Intelligent Material Systems and Structures, vol. 19, no. 3, 2008.

36 Y. Hong and A. Altman, "Streamwise Vorticity in Simple Mechanical Flapping Wings," Journal of Aircraft Vol.44, No. 5, September-October 2007 (1588-1597).

37 D. Yeo, E. Atkins, W. Shyy, “Aerodynamic Sensing as Feedback for Ornithopter Flight Control," Proc. 49 $9^{\text {th }}$ AIAA Aerospace Sciences Meeting, January 2011, Orlando, Florida. (AIAA 2011-552).

38 T. Apker, " Measurements of Time-Resolved Flapping Wing Vehicle Forces in Hover," Proc. AIAA Atmospheric Flight Mechanics Conference, 10-13 August 2009, Chicago Illinois. (AIAA 2009-5847).

39 A. Gogulapati and P. Friedmann, "Approximate Aerodynamic and Aeroelastic Modeling of Flapping Wings in Hover and Forward Flight,' Proc. 52nd AIAA Structures, Structural Dynamics and Materials Conferenc, Apr. 4-7, 2011 , Denver, CO. (AIAA-2011-2008) 